\title{
Male Partner Involvement in Promoting Antenatal Care and Skilled Delivery Attendance in Bumula Sub-County, Kenya
}

\author{
Rose A. M. Nyang'au, Tom Were, and Maximilla Wanzala
}

\section{ABSTRACT}

Globally, male partner involvement in antenatal care and skilled delivery services remains a challenge to effective management of maternal health during pregnancy. Maternal morbidities and mortalities have been increasing due to the underutilization of antenatal care and skilled delivery services at a global level. However, developed countries have had a different story on male partner involvement in antenatal care and skilled delivery services. Most male partners are involved in the process. However, SubSaharan Africa records the highest percentage of maternal morbidities and mortalities with low male partners involved in the said maternal services. Men are never active in planning for antenatal and skilled delivery services nor accompanying their female counterparts to visit antenatal clinics and maternity to receive skilled delivery. In Kenya male partner involvement in maternal services remains low despite it being recognized as one of the pillars of safe motherhood. Therefore, this study was conducted to investigate the level of male partner involvement in promoting antenatal care and skilled delivery services in Bumula Sub-County of Bungoma County, Kenya. The study adopted a descriptive cross-sectional design. The target populations were married men and community health volunteers. The study had a sample size of $\mathbf{4 2 0}$ persons. Data was collected using a pretested semi-structured questionnaire, key in-depth interview guides, focused group discussion guides and observation checklist. Data were analysed using descriptive statistics with the aid of the Statistical Package for Social Sciences version 25. Data were presented using tables, figures, narratives and direct quotes. The study found out male partner involvement was at $18 \%$, which was low in promoting antenatal care and skilled delivery services. Only $16.9 \%$ of male partners planned with their female counterparts for antenatal care and skilled delivery services. Moreover, $54.1 \%$ of male partners accompanied their female partners to the clinics and $96.5 \%$ provided support to ensure their partners accessed the services. The study concluded that there was a low level of male partner involvement in promoting antenatal care and skilled delivery attendance. The low level of male partner involvement was influenced by cultural restrictions, inadequate awareness, busy male work schedules and laxity among men. The study recommended that the two levels of government through the Ministry of Health should create awareness campaigns and public education with a targeted massage on negative cultural practices/mindset that hinder male partner involvement in maternal health services in the Bumula sub-county.

Keywords: Antenatal care, maternal health, male partner involvement, safe motherhood, skilled delivery.

\section{INTRODUCTION}

Male partner involvement in maternal health services during pregnancy is one of the foundations for safe motherhood. Looking at the developed countries such as Sweden and the United States of America, male partners are actively involved in pregnancy, which is supported by strong laws and policies [1]. In Asian countries, such as Bangladesh
Submitted : July 10, 2021

Published. : October 14, 2021

ISSN: 2593-8339

DOI: $10.24018 /$ ejmed.2021.3.5.978

R. A. M Nyang'au*

Webuye County Hospital Kenya Medical Training College, Nairobi, Kenya.

(e-mail: rosemogoi@gmail.com)

T. Were

Masinde Muliro University of Science and Technology, Kakamega, Kenya.

(e-mail: twere@mmust.ac.ke)

M. Wanzala

Masinde Muliro University of Science and Technology, Kakamega, Kenya.

(e-mail: mwanzala@mmust.ac.ke)

*Corresponding Author
$(40 \%)$ and Nepal $(57.6 \%)$ of male partners were actively involved in their partner's reproductive health services [3],[4]. In developed countries, maternal health affair is important for their population growth, which makes it possible to have an investment in good health systems. According to [5], husbands were not involved in planning for and deciding where their female partners would seek services because they felt they had no role to play in pregnancy issues. 
In Europe, male partners were jointly involved in planning for antenatal care and supported spouses emotionally during delivery [6],[7]. In Bangladesh, 47\% accompanied their wives to antenatal care and $67 \%$ during delivery [7]. In Malaysia, [8] most (89\%) male partners accompanied their female partners to attend antenatal care and about $57 \%$ to delivery. However, in Nepal, men were involved in incomegenerating activities hence lacked time to accompany their spouses for antenatal services [9]. It was found that in Pakistan there was good practice by male partners in attending antenatal care with $95 \%$ of the males accompanying their female partners [10]. In Bangladesh, male partner involvement in planning was important in supporting their wives during pregnancy, childbirth and the postpartum period because this period was perceived by male partners to be risky [11].

Developing countries have challenges in their health systems; would that be the reason why developing countries have increased maternal mortality and morbidity? In subSaharan Africa, there is still challenges of male partner involvement in maternal health. Provision of financial support and performing hard chores, arranging for transportation to health facilities and provision of medication required by the female counterparts were some of the male supports given by male partners in South Africa [12],[13]. According to [14], male partners perceived that planning for antenatal care and skilled delivery was a female responsibility and hence they were not involved. Poor spousal communication was an impeder to planning for maternal health services in Ghana [15]. Male partners involvement in antenatal care was still below the World Health Organisation threshold [16]- [19]. The weakness of these studies in SubSaharan Africa investigated only antenatal care and left skilled birth services. However, [20] reported that most (89\%) male partners were involved in planning and making a joint decision on where the partner would seek antenatal care service in Tanzania. This high involvement was attributed to the successful implementation of safe motherhood projects. In Sub-Saharan Africa countries such as Namibia, Ethiopia and Nigeria there is a low level of accompaniment with belief that pregnancy is not a role for men [21]-[23]. According to [24] in Ethiopia, men supported their spouses with household chores, arranging and buying food for them and reminding them to take medicines prescribed in antenatal care clinics. In Uganda, revealed that only $62 \%$ of male partners provided transport to their partners to access antenatal care and $73 \%$ provided nutritious food as advised [25]. After all these, male partner involvement as recorded by reviewed literature was still low.

Male partner involvement in maternal health services in Kenya in choice of the delivery site as found by [26] was still low, besides, planning with the partner on the pregnancy progress was further lower. In Mombasa County, Kenya, most of the male partners, seldom participated in planning/deciding on delivery sites and accompanied them for antenatal care and skilled delivery services [26]. In Malindi Sub-County, Kenya, [27] posited that a lack of involvement of male partners in planning maternal health services contributed to the underutilization of skilled birth services by pregnant women. However, [28] found out that in Mumias West and East, male partners were much involved in planning for services and further provided funds for antenatal care and skilled delivery. However, cultural beliefs restricted male partners from accompanying their female counterparts for these services. Reference [29] stated that male partner involvement was a determinant of female participation in pregnancy care services and influenced the decision on the timing of antenatal care visits, financial support for antenatal care visits and decision on the health care facility for delivery; however, this in Njoro, Kenya was low amongst male partners, which was attributed to tight time schedules and difficulties in getting permission from work. It was important to understand the local neighbouring studies to the study area with similar characteristics on maternal health studies. It was established from [30]in Butula sub-county of Busia County Kenya that few male partners were involved in antenatal care and skilled delivery services, but $55.8 \%$ of male partners accompanied their female counterparts to antenatal care clinics for services. This was attributed to busy schedules and cultural impeders. A Kenyan national survey on male involvement in family planning services indicated that male involvement in maternal services was associated with increased uptake of maternal health services and infant BCG immunization [31]. Therefore, why was it that Bungoma County was one of the leading Counties in Kenya still experiencing challenges in male partner involvement in antenatal care and skilled delivery services despite them being free?

In 2014, it was reported by Kenya Demographic Health Survey that $92 \%$ of pregnant women attended antenatal care services at least once, however, only ( $58 \%)$ of the expectant mothers completed the four minimum antenatal care visits and only (68\%) received skilled delivery services. To achieve Sustainable Development Goal three, aiming at reducing maternal mortality ratio (MMR) by $75 \%$, Kenya should have an MMR of 147/100,000 live births by 2030 (SDG\#3) [37]. In order to address this, faster progress is needed in the coverage of antenatal care and skilled delivery services in high burden regions. The level of male partner involvement in the said services was reported to be low but how low was not captured in most studies [32]. Furthermore, there is no data to show the level of male partner involvement in the said services This study therefore aimed at investigating the level of male partner involvement in promoting antenatal care and skilled delivery services in Bumula Sub- County, Bungoma County, Kenya.

\section{PRoblem Statement}

Bungoma County ranks among the bottom 15 counties in Kenya with a high maternal mortality rate of 384/100,000 live births. Additionally, it was ranked among the bottom fifteen counties with the lowest proportion of women receiving the minimum four recommended antenatal care visits (50\%) and skilled delivery (68\%) [33], [32]. Bumula Sub-County of Bungoma County is a rural sub-county whose only $85 \%$ of pregnant women complete the 1st antenatal care services while $30 \%$ complete the four minimum visits and only $47 \%$ received skilled delivery [34]. There is a paucity of information on the level of male partner involvement during pregnancy as there is no documentation. This makes it 
difficult to determine the level of male partner involvement. Despite the growing evidence on the benefits of engaging male partners in maternal wellbeing programmes, their participation in Bumula is not known. At the community level, community health volunteers ( $\mathrm{CHVs}$ ) refer women with their partners for antenatal care and skilled delivery services but there is also no data to show the number of male partners reached. It is against this backdrop that this study sought to investigate male partner involvement in promoting antenatal care and skilled delivery attendance in Bumula SubCounty, Kenya.

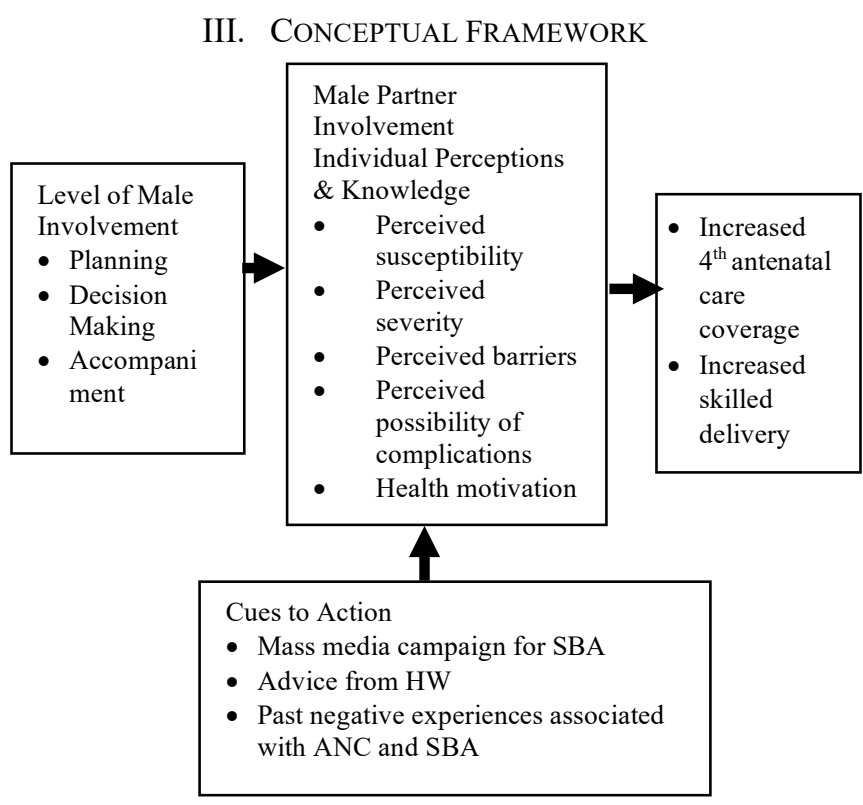

Fig. 1. Conceptual framework.

\section{Materials And Methods}

The study employed a descriptive cross-sectional research design. The design was flexible in describing the level of male partner involvement in promoting antenatal care and skilled delivery attendance. The study area was Bumula subcounty with a focus on the level of male partner involvement in promoting antenatal care and skilled delivery attendance. The study targeted male partners with a child of 12 months or less. Key informants comprised community health volunteers (CHVs) and were used to corroborate the findings from the male partners in the study. The study area had a target population of 9,525. Simple random sampling was used to determine the sample size for this study whose data was collected using a structured questionnaire. Purposive sampling was used to select key informants who were interviewed to shed more light on the findings from quantitative data from questionnaires. The level of male partner involvement in promoting antenatal care and skilled delivery attendance was obtained. Research assistants were recruited and trained for three days and they assisted in data collection.

The collected data were coded and entered into analysed software - Statistical package for social sciences (SPSS) version 25. Descriptive statistics were presented in tables in form of proportions. Key informant data were reported in narration format. Ethical clearance was obtained from the Institutional Ethics and Review Committee of Masinde Muliro University of Science and Technology and the National Commission for Science, Technology, and Innovation. The researcher maintained the integrity and privacy of the participants.

\section{RESUlts}

It was established by this study that only $18 \%$ of the male partners were involved in promoting antenatal care and skilled delivery attendance in Bumula sub-county Kenya. The results illustrated in Fig. 1, shows the level of male partner involvement in promoting antenatal care and skilled delivery attendance. Male partner involvement was measured at three levels: male partner involved in planning for antenatal care and skilled delivery services of his partner, accompanying partner to the health facility for antenatal care and skilled delivery attendance services and provision of support to his partner to access antenatal care and delivery services. The three levels were collapsed and measured as one variable: level of male partner involvement. The findings showed that only $18 \%$ of the male partners in Bumula Sub-county of Bungoma County were involved in promoting antenatal care and skilled delivery attendance. Some of the key informant and focus group discussion participants' responses were in agreement with these findings. A majority of the Key Informants $85.7 \%$ (six out of seven) reported a low level of male partner involvement as illustrated below:

1. ... Male partner involvement in antenatal care services of their female partners is very poor but slightly better during the delivery period.

2. ... since male partner involvement is low, during our household visits, we normally remind and encourage men on the need for their involvement in care and support of their pregnant partners but most of them are reluctant and others are busy with their daily working schedules.

Equally, 77\% (17/22) of the male partners in focus group discussion reported that there was a general low male partner involvement in antenatal and skilled delivery services. They added that men were usually involved in providing support at the household level in cases where the women are unable to support themselves as summarized by the following quote:

1. ...men in this community rarely involve themselves in pregnancy and childbirth activities. They are restricted by the culture which does not permit them to be actively involved in issues to do with pregnancy and child-birth.

Male partner Involvement in promoting ANC

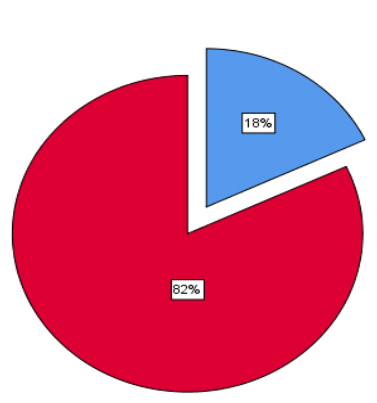

Fig. 1. Male partner involvement in promoting antenatal care and skilled delivery services. 
According to Table I, $16.9 \%$ of the male partners were involved in planning for antenatal care and skilled delivery; about $54.1 \%$ accompanied their partners to the health facility; $96.5 \%$ provided support in various forms such as $26.5 \%$ monitored nutritional requirements of their partners, $23.8 \%$ supported household chores, 20.3\% supported with birth preparedness, $19.1 \%$ provided financial support and $6.8 \%$ provided psychosocial support. The findings in Table I were in agreement with the discussions with men during focus group discussion.

It was ascertained by 8 out of $22(36 \%)$ of the focus group discussion participants that it was common for men to plan together with their partners as explained in the following quotes:

1. ... It was my first child and thus it was important for me to be part of the planning process. Naturally, it is a woman's responsibility but I had to invest my time in her pregnancy.

2. ... My wife was sickly before I realised that she was expectant. This made me ask her to attend clinics to diagnose her condition.

3. ... My wife had only one child and that was not enough according to our family plan. We tried to get another. So, it was of utmost importance for her and me to plan together ad attend clinics to procure guidance on our future possibilities of the unforeseen child.

The $64 \%$ (14 out of 22) of the men who had a different opinion regarding planning, explained that planning was uncommon because most male partners assumed that once the women realised they were expectant or they were experiencing labour pains they knew what would happen next. According to them, this did not require their involvement.

1. ... My wife avoided sharing any information regarding pregnancy or pregnancy-related conditions. She seldom told me about her hospital visits and the purposes that drove her to the clinic.

2. ... Women know about their welfare - health-wise or otherwise - therefore, as the man in the house I have nothing to offer. She is the professional one in the house with a wealth of knowledge in maternal issues.

According to the Key Informants, the current birth plan emphasises planning for delivery leaving out planning for antenatal care services. According to most of them $71 \%$ (five out of seven), reported that this could be contributing to inadequate involvement as mentioned by one Key informant:

1. ...We usually sensitise men to be involved in planning for skilled delivery. There is little emphasis on planning for antenatal care services. I feel we should have a plan for antenatal care services.

According to the focus group discussion forum, about accompaniment, $45.5 \% \quad(10 / 22)$ of male partners accompanied their partners to antenatal care/ skilled delivery. However, this was contradicted with the findings in Table I which indicated $54.1 \%$ accompaniment.:

Reasons for Accompanying Included:

1. ...My partner was very weak and sickly during her last pregnancy. It was my role as her husband to accompany her to the clinic and even explain to the health worker how my wife felts. Leaving her alone to get to the clinic could be dangerous.
2. ...I accompanied her once because the nurse had advised her to do so during her next visit. She had complications that could not be handled in Siboti. So I was invited to be explained to.

3. ... She developed labour pains before her delivery time, I had to seek help immediately from the hospital.

4. ... These days the government has really helped us! It's doing everything, even buying few items for the baby. We are now focusing on other things, nothing is left for us to be involved!

\section{Reasons for not accompanying:}

Conversely, $55 \%(12 / 22)$ of male partners in the focus group discussion did not accompany their female partners and the following number of reasons.

1. ... I didn't accompany her because I didn't know it was a requirement and after all our culture does not permit us. However, my brother's wife accompanied her for delivery.

2. ... Most men in this community don't accompany their wives for clinic services, but few do for deliveries especially when their mothers and co-wives are not around. The delivery period is dangerous. They have to accompany them because in case anything bad happens they don't get blamed. The delivery period is not a joke! Women undergo difficulties and need to be supported properly.

3. ... To my knowledge, pregnancy is not a disease! I didn't see why she needed to be accompanied. I left her to go alone while I went to work. During delivery, our CHV escorted her to the hospital. I followed her later after she has delivered.

4. ... It's better to speak the truth here, some of us are guilty of not accompanying the partners to the health facility for antenatal care or delivery services because of previous negative events that we encountered.

The reasons for accompanying or not accompanying female partners for services also were in line with the reasons articulated by Key Informants who explained that:

1. ... As CHVs, we refer men with their partners for antenatal care services to the nearest health facilities. Some of those who accompany their partners, come back complaining that they were left unattended to, so they never see the need to be there hence they won't accompany their partners during the next visit.

2. ... Sometimes back we used to have men to men support groups in each sub-location where men were given education on their roles during the pregnancy period of their partners. During that time, men accompanied their partners for services, but since the NGO that supported the program left, the support groups just died. Very few men nowadays accompany their partners.

3. ... Some men have left their responsibilities to us CHVs especially when their partners have pregnancyrelated complications. We refer them to the health facilities without their presence.

Additionally, findings from focus group discussion on other support male partners provided to their female partners to ensure they sought antenatal care and skilled delivery services corroborated with findings in Table I as presented herein. 


\section{A. Financial Support}

1. ...When my wife told me, she was going to the hospital for the antenatal care clinic, I provided her with money for transport.

2. ... I bought items for delivery and saved some money to spend during the delivery time in case of emergency.

3. ... I bought medicines that were prescribed for her use and also paid for an X-ray that the doctor had advised her to do.

4. ... I hired a house help to assist her during her last pregnancy. She once lost a pregnancy because of overworking. I had to be careful this time around.

B. Physical and Psychological Support

... During the last pregnancy of my wife, I requested my sister to assist her with household chores because I was always out.

... For me, I was always out by $0500 \mathrm{~h}$. When I returned in the evening, I ensured I had carried enough food and fruits for her.

... My wife was sickly and weak during her last pregnancy, I also kept on encouraging her that all will be well.

... Malaria disturbed her, I ensured that she slept under a mosquito net, took her medicines as advised at the clinic and I ensured she got to the clinic when the dates were due.

Furthermore, some of the findings from the direct observation made in the visited health facilities also were in agreement with findings in Table I in all the seven facilities that were visited, few male partners were observed accompanying their partners for antenatal care services; however, they kept themselves away from the clinic environment. A few of them were seated under shades waiting for their partners as they received services. Others were found at the postnatal unit visiting their partners postdelivery while others were waiting from outside while their partners received services in labour wards.

TABLE I: LEVEL OF MALE PARTNER INVOLVEMENT IN PROMOTING ANTENATAL CARE AND DELIVERY ATTENDANCE

\begin{tabular}{lc}
\multicolumn{2}{c}{ ANTENATAL CARE AND DELIVERY ATTENDANCE } \\
\hline \hline \multicolumn{1}{c}{ Level of male involvement } & Percent (\%) \\
\hline \hline Planned for antenatal care with the partner & \\
(where \&when) & 16.9 \\
Yes & 83.1 \\
No & \\
Accompanied the partner to the clinic for services & 54.1 \\
Yes & 45.9 \\
No & \\
Supported the partner & 23.8 \\
Household chores & 6.8 \\
Psychosocial & 20.3 \\
Birth preparedness & 26.5 \\
Monitoring nutritional requirements & 19.1 \\
Financial & 3.5 \\
No support &
\end{tabular}

[Results presented in proportion (\%); $n, 344]$.

The study also established that the majority $(82 \%)$ of male partners were not involved in promoting antenatal care and skilled delivery service. Even though it was not within the scope of the study, it was prudent to find out the reasons that contributed to their lack of involvement. The study established that cultural restrictions contributed $43.3 \%$ of the male non-involvement in antenatal care and skilled delivery services. Also, there were other intrinsic contributors to the non-involvement of male partners in antenatal care and skilled delivery attendance and were: the lack of awareness (24.4\%), other family members taking up the role of the male partner $(16.3 \%)$, inherent perception $(11.6 \%)$, peer influence $(2.3 \%)$ and partners living away from home $(2 \%)$. This reason $\mathrm{s}$ stood out in the study that was worth mentioning to understand areas that need addressing to improve on male partner involvement in antenatal care and skilled delivery attendance. The results were illustrated in Table II.

TABLE II: REASONS FOR THE NON-INVOLVEMENT OF MALE PARTNERS IN PROMOTING ANC AND SKILLED DELIVERY ATTENDANCE

\begin{tabular}{cc}
\hline \hline Reason & Percent (\%) \\
\hline \hline Cultural restrictions & 43.3 \\
Living away from home & 2.1 \\
Other family members taking & 16.3 \\
up the male partners' roles & 11.6 \\
Inherent perception & 2.3 \\
Peer influence & 24.4 \\
Not aware I should be involved & \\
in ANC and delivery & \\
\hline \hline
\end{tabular}

The results were presented proportions (\%); n, 344.

Male partner involvement is the epitome of safe motherhood globally. While this is known, about $68 \%$ of male partners who participated in this study noted that there had not been adequately involved in antenatal care and skilled delivery services of their female partners. However, a majority of them $86 \%$ agreed that adequate involvement would increase the uptake of the said services by their female counterparts as described in Table III.

TABle III: MALE PARTNERS' OPINIONS ON THE LEVEL OF MALE PARTNER INVOLVEMENT

\begin{tabular}{|c|c|c|}
\hline Question & Yes & No \\
\hline $\begin{array}{c}\text { Were you adequately } \\
\text { involved in your partner's last } \\
\text { pregnancy to ensure that she } \\
\text { utilized all antenatal care and } \\
\text { delivery services she required } \\
\text { for her? }\end{array}$ & $32 \%$ & $68 \%$ \\
\hline $\begin{array}{l}\text { In your own opinion, do you } \\
\text { think adequate male partner } \\
\text { involvement in maternal } \\
\text { services can promote the } \\
\text { utilization of antenatal care } \\
\text { and skilled delivery services } \\
\text { by their partners? }\end{array}$ & $86 \%$ & $14 \%$ \\
\hline
\end{tabular}

The study findings above established that there was low male partner involvement in promoting antenatal care and skilled delivery attendance in the Bumula sub-county. It further established from male participants and Key informants what needed to increase the level of male partners' involvement in order to promote antenatal care and skilled delivery services of the female partners. Responses were themes as shown below:

Increase Public Awareness on the Need for Male Involvement:

1. ... $\mathrm{MOH}$ should conduct public awareness with targeted information for men, to reinforce and emphasize the importance of male partner involvement ANC and delivery period.

2. ...What makes us not accompany our partners to the clinic is that the health care workers don't insist that 
we should accompany them. Hence, it's not a big issue to bother ourselves to get to the clinic. If they want us to be involved, let them emphasize this in a manner that can be taken seriously.

3. ...I suggest that during chief baraza's day besides discussing issues of that day, we should have a specialist to keep reminding men on the importance of male involvement.

Even if we accompanied our spouses, we cannot do it all the time, it will be better to be told at what level are we supposed to accompany them.

Change of male mindset on the need to be involved:

1. ...It's high time for us men to be serious on this matter and get involved as required. There is no excuse for our pregnant women not attending antenatal care clinics as required because of us, the services are here with us, let's use them.

2. ...Let the truth be said, some of us are reluctant to get to the clinic with our partners because of previous negative experiences.....not all that happened previously is happening now.

Disregard negative cultural beliefs on male partner involvement in maternal health services:

1. ...It's difficult to change what people are used to be doing, but slowly with frequent reminders, they can disregard these negative cultural practices.

2. ...Presently, our culture is fading, people don't nowadays strictly follow what the culture dictates, though there are those few who cannot be involved in activities concerning women and pregnancy.

Similar suggestions were also shared by most Key informants. However, one key informant had this different suggestion:

1. ...There is no uniformity in the manner in which male partner involvement in maternal health services is advocated for and implemented. We work at the community level, implement what we have been directed to do. For us, it was an NGO's program, now that the NGO's contract came to an end, the program also died. The MOH didn't continue with what the NGO initiated, there should be uniformity in the manner in which the $\mathrm{MOH}$ and partners work to avoid confusion.

Of the $82 \%$ of the non-involved male partners, it was reported that the male partners were restricted by cultural beliefs (43.3\%), lack of awareness $(24.4 \%)$, other family members took up the role of the male partner $(16.3 \%)$, inherent perception $(11.6 \%)$, peer influence $(2.3 \%)$ and partners were living away from home $(2 \%)$. It was also outstanding that most $(68 \%)$ of female partners failed to adequately involve ale partners in the pregnancy process. It was known to most (86\%) male partners that their involvement in antenatal care and skilled delivery attendance would promote the utilisation of antenatal care and skilled delivery attendance.

The participants in a Focused Group Discussion (FGD) forum $(36 \%)$ stated that it was important for men to plan together with their partners. About $45 \%$ of these participants accompanied their female partners to antenatal care clinics and skilled delivery. The $45 \%$ of the participants observed that the partner at the time was very weak and sickly, which a husband it was his role to accompany her to the clinic and even explain to the health worker how she was feeling. For some, it was a requirement for the last visit for him to be present. Labour pains were some of the precursors that necessitated the accompaniment. It was observed from the $55 \%$ of the male partners who failed to accompany their wives that they were bound by the cultural belief that did not permit them to accompany their female partners to antenatal care or skilled delivery. Some stated that pregnancy was not a disease thus it was not in his place to be present at the facility.

Support was vital as indicated by $96.5 \%$ of the male partners supported their female counterparts. Financially, pregnant women required support thus money is required to facilitated transportation, purchase baby stuff, medication, for hired assistance at the house. Physical and psychological support was essential for a better obstetric outcome; therefore, it was important for male partners to help with household chores and provide a safe nesting place for her.

Key informants (Community Health Volunteer - CHVs) stated that:

1. ... During our household visits, we normally remind and encourage men on the need for their involvement in care and support of their pregnant partners but most of them are reluctant and others are busy with their daily working schedules.

2. ... Us as CHVs, we refer men with their partners for antenatal care services to the nearest health facilities. Some of those who accompany their partners, come back complaining that they were left unattended to, so they never see need to be there hence they won't accompany their partners during the next visit.

3. ... Sometimes back we used to have men to men support groups in each sub-location where men were given education on their roles during the pregnancy period of their partners. During that time, men accompanied their partners for services, but since the non-governmental organisation (NGO) that supported the program left, the support groups just died. Very few men nowadays accompany their partners.

4. ... Some men have left their responsibilities to us CHVs especially when their partners have pregnancy-related complications. We refer them to the health facilities without their presence.

\section{Discussion}

The study recorded a low level $(18 \%)$ of male partner involvement in promoting antenatal care and skilled delivery. Male partner involvement was measured at three levels; planning and decision making, accompaniment, and provision of support. These results are reflective of previous studies conducted elsewhere in Kenya [26]-[29]. However, unlike this study, these previous studies did not measure male partner involvement at the three levels as presented in this study. Again, they focused on antenatal care and uptake of skilled delivery in isolation. The results were lower compared to studies from other sub-Saharan Africa countries particularly Ghana and Ethiopia which recorded (67.2\%) and (41.4\%) respectively [18], [17]. Unlike this study, [17] targeted female respondents. The difference observed could 
be due to a difference in methods employed to construct the involvement level as well as the study setting. For example, previous studies were hospital-based while the current study was community-based. These results contrasted with those recorded from studies conducted in Sweden, America and Nepal where the majority of the male partners were involved in their partner's reproductive health services. According to [1], [2], most males who were involved were first-time fathers and it was anchored in legislation. Most participants in this study were not first-time fathers (had more than two children). Although it's a policy in Kenya for males partners to be involved in antenatal care and skilled delivery services, most respondents indicated that culture restricted them.

It is within the safe motherhood and Sustainable Development Goal number three for partners (male and female) to plan together and decide where to seek maternal services from. Surprisingly, this was not the case for Bumula Sub-County. The results showed that only $16.9 \%$ of the male partners participated in planning and decision-making on antenatal care and skilled delivery attendance. The respondents indicated their expectant spouses already perceived what was expected of them regarding attending antenatal care and skilled delivery. This could explain why there is very low coverage of antenatal care and skilled delivery services in Bumula sub-county. The men in Bumula have left pregnancy issues to their female counterparts who alone were unable to manage the process as required. This result contrast sharply with [20] and [28] who established that majority of male partners were involved in where their partners would seek antenatal care services.

According to [28], culture influenced male partners involved which were in support of this study attributed low level of male partners participation to cultural barriers and norms which require other female family members to take up the male partners roles during the pregnancy of their spouses. According to a Focused Group discussion (FGD) forum, it was stated by a member that culturally the Bukusu men had no role to play during their female partner's antenatal care and skilled delivery attendance). Basing on the FGD findings, the researcher concurred with the findings that Bumula subcounty was mostly occupied by the Bukusu sub-tribe of the Luhya tribe, which they hold dearly on the cultural norms and taboos. Besides, male partner involvement in most cases was delegated to female partner's relatives such as the mother-inlaw and female relatives from both families (husband and wife). The role of men, therefore, stopped at providing upkeep for the family.

It was also discovered that most families in the Bumula sub-county perceived pregnancy as a normal process, not a health complication, one that the wife comfortably could manage. This was supported by the key informant interview that suggested that most women from the Bukusu tribe did not permit male partner involvement where they failed to disclose when they got pregnant and when they began attending antenatal care clinics, which hampered male partner involvement. However, this study was in contradiction with other scholarly works of [28] in Kenya and [20] in Tanzania that male partners participated in planning for antenatal care and skilled delivery attendance and [8] in the Muar district that reported $91.3 \%$ male involvement in the planning phase. The inconsistencies might have been caused by the set-up of those studies where in most cases those study areas had enough primary health care centres that were accessible and available. The health workers were locals. The awareness levels among male partners were high with most printed materials on the importance of male partners' involvement.

This study found out that most $(54.1 \%)$ male partners in the Bumula sub-county accompanied their female partners to attend antenatal care and skilled delivery at least once. Most of the male partner's accompaniment was need driven. The majority of them were motorbike riders, so they provided transport to their partners. This was in agreement with what was observed from the facilities visited. The male partners dropped their spouses at the clinics hanged around and waited for them to receive the services. No man was found at the waiting bays or in the consultation rooms with their partners. From the Focused Group Discussion forum, it was stated that male partners accompanied their female counterparts to antenatal care and skilled delivery. These findings agree with those of [28] who reported that despite cultural barriers, most men accompanied their spouses to antenatal care. Accompanying the female partner to the health facility for antenatal services and skilled delivery is a key factor in determining the level of uptake and adherence to the programmes. WHO recommends that an expectant mother attends antenatal care earliest in pregnancy and continues utilizing these services up to six months post-delivery. Female partners who are accompanied to the clinics with their male partners are more likely to follow the schedules up to 6 months after delivery [35]. Looking at [20] in Tanzania, about $63.4 \%$ of male partners accompanied, [7] in Bangladesh who reported accompaniment at $89 \%$ of partner to antenatal care and $57 \%$ during delivery, [8] who reported $95 \%$ accompaniment in Malaysia and [36] in Rwanda, most (86.8\%) male partners accompanied female partners to antenatal care, there is a positive trajectory towards increased accompaniment among male partners.

The majority (96.5\%) of male partners provided support to their female partners during antenatal care and skilled delivery services. However, the level of male involvement still remained low. According to key informant interviews, most male partners were active in the provision of psychosocial and financial support. This kind of support encouraged and empowered female partners during antenatal care and skilled delivery attendance. This study was in agreement with [27] study in Malindi sub-county $83 \%$ of male partners were involved and [28] study in Mumias East and Mumias West sub-counties where $69.7 \%$ of male partners provided financial support. Besides, this was also a true reflection of a study by [15] findings which noted that about $96.5 \%$ of male partners provided financial support for ANC services.

It is well known that the male partner is the head of the family, therefore, should lead the family from the frontline. Key informants stated that as health workers they were keen on encouraging male partners' active involvement in the care and support of pregnant mothers during the antenatal and skilled delivery period. However, some male partners delegated their support to community health volunteers (CHVs) to see through pregnancy-related complications. The assumption being that the CHVs knew about what pregnancy demands at the time and they would be of better assistance as 
compared to male partners. It becomes quite disturbing when the man fails to perceive pregnancy as a delicate period, which key informant interviews stated that male partners in Bumula sub-county did not treat pregnancy as risky. This study found out that male partners in Bumula sub-county supported their female partners during antenatal care and skilled delivery, whose finding was in support of [13].

\section{CONCLUSION}

There was a low level of male partner involvement in antenatal care and skilled delivery attendance. About $18 \%$ of male partners were involved in antenatal care and skilled delivery attendance. There was the improvement of male partner involvement in accompaniment and provision of support (finances, help with household chores and birth preparedness) that met antenatal care and skilled delivery requirements. Most of the male partners in the Bumula subcounty attached a lot of health risks to delivery and were therefore mostly involved during that time.

\section{RECOMMENDATION}

The two levels of government through the Ministry of Health should create awareness campaigns and public education with a targeted massage on negative cultural practices/mindset that hinder male partner involvement in maternal health services in the Bumula sub-county. Kenya Ministry of Health should assess, review and strengthen the policy on male involvement in maternal services to ensure acceptable full implementation to promote male partners involvement in antenatal care and skilled delivery attendance.

\section{ACKNOWLEDGMENT}

First of all, I would like to thank the Almighty God for the gift of life and love for me to pursue this postgraduate degree. I acknowledge the guidance and support from my supervisors Dr Tom Were $(\mathrm{PhD})$ and Dr Maxmilla Wanzala $(\mathrm{PhD})$ throughout this thesis development. I acknowledge the support from my classmates during the development of this thesis. Likewise, I confer my gratitude to my research assistants Mr Paul Kem Wafula and Mr Eugene Mwavali Amboga for their efforts and time in making this thesis a success. Finally, my vehement gratitude goes to my family Dr George Marwa (husband), Martin Luther (son), Sonya Carson and Rosa Park (daughters) for their sacrifice and patience throughout this journey.

\section{REFERENCES}

[1] E. Andersson, A. Norman, C. Kanlinder and L. Plantin, "What do expectant fathers expect of antenatal care in Sweden? A cross-sectional study," Sexual \& Reproductive Healthcare 1;9:27-34, 2016.

[2] M. Mackert, M. Guadagno, A. Lazard, E. Donovan, A. Rochlen, A. Garcia, M. J. Damásio and B. Crook, "Engaging men in prenatal health via eHealth: findings from a national survey," JMIR Pediatrics and Parenting 1(2): e7, 2018.

[3] G. Bishwajit, S. Tang, S. Yaya, S. Ide, H. Fu, M. Wang, Z. He, F. Da and Z. Feng, "Factors associated with male involvement in reproductive care in Bangladesh," BMC Public Health 17(1):1-8, 2017.

[4] C. K. Bhusal and S. Bhattarai, "Social Factors Associated with Involvement of Husband in Birth Preparedness Plan and Complication
Readiness in Dang District, Nepal," Journal of Community Medicine \& Health Education 8(06), 2018.

[5] S. Lewis, A. Lee and P. Simkhada, "The role of husbands in maternal health and safe childbirth in rural Nepal: a qualitative study," $B M C$ Pregnancy and Childbirth 15(1):1-0, 2015.

[6] A. Ghimire and K. Pun, "Male Involvement in Maternal and Infant Health Care, Banke, Nepal," International Journal of Nursing Research and Practice 4, 2017.

[7] A. E. Rahman, J. Perkins, S. Islam, A. B. Siddique, M. Moinuddin, M. R. Anwar, T. Mazumder, A. Ansar, M. M. Rahman, S. Raihana and C. Capello, "Knowledge and involvement of husbands in maternal and newborn health in rural Bangladesh," BMC Pregnancy and Childbirth 18(1):1-2, 2018.

[8] R. R. Marzo, A. Ahmad, G. V. Xit, N. C. Ming, M. T. Win, T. Talukder and J. R. Naidu, "A study of involvement in maternal health by male counter-part in the family in Muar district," 2018.

[9] S. Sharma, K. C. Bhuvan and A. Khatri, "Factors influencing male participation in reproductive health: a qualitative study," Journal of Multidisciplinary Healthcare 11:601, 2018.

[10] M. Younas, Y. Parpio, T. Saeed Ali and S. Awan, "Male partners' knowledge and practices of antenatal care in district Swat, Khyber Pakhtunkhwa, Pakistan: A cross-sectional study," Journal of Midwifery and Reproductive Health 1;8(1):2005-15, 2020.

[11] M. Zakaria, A. K. Khan, M. Ahmad, F. Cheng and Xu J. "Women's Perception of Male Involvement in Antenatal, Childbirth and Postnatal Care in Urban Slum Areas in Bangladesh: A Community-Based CrossSectional Study," Healthcare 9(4):473,2021.

[12] K. Nesane, S. M. Maputle and H.Shilubane, "Male partners' views of involvement in maternal healthcare services at Makhado Municipality clinics, Limpopo Province, South Africa," African Journal of Primary Health Care and Family Medicine. 1;8(2):1-5, 2016.

[13] J. A. Greenspan, J. J. Chebet, R. Mpembeni, I. Mosha, M. Mpunga, P. J. Winch, J. Killewo, A. H. Baqui and S. A. McMahon, "Men's roles in care seeking for maternal and newborn health: a qualitative study applying the three delays model to male involvement in Morogoro Region, Tanzania," BMC Pregnancy and Childbirth 19(1):1-2, 2019.

[14] M. G. Matseke, R. A. Ruiter, V. J. Rodriguez, K. Peltzer, G. Setswe, S. Sifunda, "Factors associated with male partner involvement in programs for the prevention of mother-to-child transmission of HIV in rural South Africa," International Journal of Environmental Research and Public Health 14(11):1333, 2017.

[15] J. P. Craymah, R. K. Oppong and D. A. Tuoyire, "Male involvement in maternal health care at Anomabo, central region, Ghana," International Journal of Reproductive Medicine 2017.

[16] R. A. Aborigo, D. D. Reidpath, A. R. Oduro and P. Allotey, "Male involvement in maternal health: perspectives of opinion leaders," $B M C$ Pregnancy and Childbirth 18(1):1-0, 2018.

[17] F. Kassahun, C. Worku, A. Nigussie and G. Ganfurie, "Prevalence of male attendance and associated factors at their partners antenatal visits among antenatal care attendees in Bale Zone, South East Ethiopia," International Journal of Nursing and Midwifery 10(9):109-20, 2018.

[18] M. T. Kumbeni, F. A. Ziba, J. N. Alem and S. A. Nborah, "Factors Influencing Male Involvement in Antenatal Care in the Kassena Nankana Municipal in the Upper East Region, Ghana," European Scientific Journal 15:21, 2019.

[19] C. J. Mbadugha, C. J. Anetekhai, A. L. Obiekwu, I. Okonkwo, J. A. Ingwu, "Adult male involvement in maternity care in Enugu State, Nigeria: A cross-sectional study," European Journal of Midwifery 3 , 2019.

[20] N. S. Gibore, M. J. Ezekiel, A. Meremo, M. J. Munyogwa and S. M. Kibusi, "Determinants of men's involvement in maternity care in Dodoma Region, Central Tanzania,” Journal of Pregnancy. 2;2019.

[21] N. Shiyagaya Karolina, S. K. Kuna and A. H. Justus, "Level of male involvement and factors influencing involvement in the prevention of mother-to-child transmission of HIV in the Oshakati, Oshana Region (Doctoral dissertation, University of Namibia)" 2016.

[22] B. H. Mohammed, J. M. Johnston, D. Vackova, S. M. Hassen and H. $\mathrm{Yi}$, "The role of the male partner in the utilization of maternal health care services in Ethiopia: a community-based couple study," $B M C$ Pregnancy and Childbirth. 19(1):1-9, 2019.

[23] O. Falade-Fatila and A. M. Adebayo, "Male partners' involvement in pregnancy-related care among married men in Ibadan, Nigeria," Reproductive Health 17(1):1-2, 2020.

[24] A. Nigusie, T. Azale and M. Yitayal, "Institutional delivery service utilization and associated factors in Ethiopia: a systematic review and META-analysis," BMC Pregnancy and Childbirth 20(1):1-25, 2020.

[25] P. Alupo, E. R. Atim, H. Kaggwa, C. Mudondo, C. Ogallo, J. Ogwang, B. Wanume, R. Nekaka and J. Nteziyaremye, "Male Partner Involvement in The Utilization of Antenatal Care Services in Kidera, 
Buyende District, Uganda: Cross-Sectional Mixed Methods Study," 2020.

[26] J. M. Onchong'a, T. Were and J. O. Osero, "Knowledge, perception and level of male partner involvement in the choice of the delivery site among couples at coast level five hospital, Mombasa County, Kenya," African Journal of Reproductive Health 20(1):71-9, 2016.

[27] L. N. Nyandieka, M. K. Njeru, Z. Ng'ang'a, E. Echoka and Y. Kombe, "Male involvement in maternal health planning key to utilization of skilled birth services in Malindi Subcounty, Kenya," Advances in Public Health, 2016.

[28] S. Kiptoo, C. Mutai and M. Kipmerewo, "Male partner involvement on maternal care services during the perinatal period in Mumias east and west sub-counties, Kakamega County, Kenya," International Journal of Advanced Research 4(11):1219-32, 2016.

[29] B. F. Rotich, R. A. Aura-Odhiambo and S. Muthoka, "The Influence of Male Spouse Participation in Maternal Utilisation of Antenatal Care in Njoro Town, Nakuru County, Kenya," 2019.

[30] F. K. Ongolly and S. A. Bukachi, "Barriers to men's involvement in antenatal and postnatal care in Butula, western Kenya," African Journal of Primary Health Care \& Family Medicine 11(1):1-7, 2019.

[31] B. Odeny, C. J. McGrath, A. Langat, J. Pintye, B. Singa, J. Kinuthia, A. Katana and G. John-Stewart, "Male partner antenatal clinic attendance is associated with increased uptake of maternal health services and infant BCG immunization: a national survey in Kenya," BMC Pregnancy and Childbirth 19(1):1-9, 2019.

[32] Kenya Demographic Health Survey (KDHS), Kenya National Bureau of Statistics (KNBS) and ICF Macro, Kenya Demographic and Health Survey, 2014.

[33] The Republic of Kenya, National Survey on male involvement in family planning and reproductive health in Kenya, National Council of Population and Development 2014, Government Printers 2014.

[34] Ministry of Health of Bungoma County, County Health Records and Information Office, 2016.

[35] World Health Organization, A practical guide to implementing safe motherhood in countries. WHO/FHE/MSM/2012.11. Safe motherhood programme. Geneva. UNICEF. Maternal mortality in 2013: Estimates developed by WHO, UNICEF, UNFPA, and the World Bank. Geneva.

[36] L. Jennings, M. Na, M. Cherewick, M. Hindin, B. Mullany, S. Ahmed, "Women's empowerment and male involvement in antenatal care: analyses of Demographic and Health Surveys (DHS) in selected African countries," BMC Pregnancy and Childbirth 14(1):1-1, 2014.

[37] D. M. Nyangena, "Infleunce of provision of free maternal health care program on maternal mortality rates in Kisii County, Kenya," International Journal of Research and Innovation in Social Science (IJRISS) |Volume IV,2020

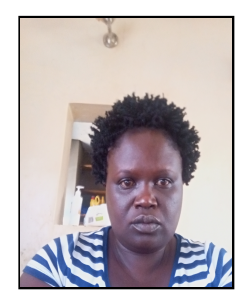

Rose A. M. Nyang'au is a Clinical Medicine Practitioner at Webuye County Hospital and a parttime Medical Tutor at Kenya Medical Training College Chwele. 